\title{
Quality control of thyroid function tests in vitro
}

\author{
A. M. BOLD AND D. M. BROWNING \\ From the Clinical Chemistry Department, Queen Elizabeth Hospital, Birmingham
}

The most sensitive method of testing whether or not a laboratory determination is in control is by the use of daily means, running daily means, and cusum techniques (Whitehead and Morris, 1969). Experience in our own laboratory and elsewhere shows that, using these methods, early loss of analytical control can be detected before other measurements, such as values on pooled sera or control sera, fall outside acceptable limits. These techniques unfortunately require large batch numbers and preferably a large proportion of 'normal' values. For tests of thyroid function in vitro most laboratories, apart from a few centres, probably have workloads that make these methods of assessing control unsatisfactory; furthermore, the tests are generally performed only on selected patients. Most laboratories, therefore, are thrown back on the older methods. In our own laboratory we have tried daily means, but in practice have to rely on analysis of a pooled serum, on commercial control sera, and on repeats of specimens from the previous run. These test reproducibility reasonably well, but are not satisfactory for accuracy. Our experience of control sera, especially for thyroxine and serum uptake tests, is that the values quoted are generally standardized by methods different from those we use. The Wellcome quality control scheme we find helpful for protein-binding iodine though thyroxine itself and serum uptake tests are not yet included in their scheme ${ }^{1}$.

\section{Survey}

In view of these problems we thought that it would be of interest to find out through the mechanism of the National Quality Control Scheme (Whitehead, Browning, and Gregory, 1973) how laboratories in this country performed the commoner thyroid function tests in vitro. There is little doubt that a challenge to determine samples completely 'blind' is much more testing than routine assay of commercial sera. Because of possible problems of stability of thyroxine in the post we decided to use commercial dried sera. Accordingly, after a preliminary enquiry to find out which laboratories were interested, in

${ }^{1}$ Thyroxine has recently been included in the Wellcome quality control scheme.
September 1972 we circulated three different speci- $-\stackrel{\circ}{-}$ mens of serum from a single batch to over $120 \vec{\omega}$ laboratories (a few laboratories receiving only? serum 1 and serum 2) and 114 laboratories sent in? returns. The returns are not necessarily completely ${ }_{\infty}$ representative, since there are undoubtedly several $\omega$ laboratories in the country, such as medical physics laboratories, which perform thyroid function tests ${ }_{\circ}^{+}$

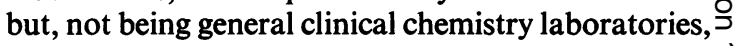
were not in the National Quality Control Scheme. An explanatory circular was sent with the sera@ together with a questionnaire in two parts (fig 1) $\stackrel{\widetilde{\partial}}{\supset}$ asking for (1) observed values, with units and $\vec{\varphi}$ method, and (2) interpretation on a five-point $\varphi$ scale.

\section{Results}

The first interesting conclusion from the survey was气 the number of laboratories measuring thyroxine $\stackrel{\mathbb{Q}}{\mathbb{2}}$ instead of, or occasionally in addition to, protein- $\overrightarrow{\overrightarrow{0}}$ bound iodine (PBI). The number of laboratories 3 using each test is indicated in table I. One or two of the kit methods had only recently become available and may soon be more widely employed.

These figures do not indicate numbers of assays? performed, and it may be that because centralized $\frac{5}{3}$ laboratories perform large numbers of PBIs, more PBIs are determined than any other test of thyroido function in vitro. Nevertheless the availability of kits for measuring thyroxine is changing the situations dramatically. Of the 114 laboratories in the survey most (79) performed thyroxine assay, Thyopac-4․ being the most widely used kit (44); 58 laboratoriesN combined a serum uptake test with PBI or thyroxine From the information received, no more than eighto laboratories (and possibly fewer, since three labora- $-\omega$ tories merely indicated that they used a competitive protein binding (CPB) method without furthero details) assayed thyroxine without a kit. Seventyd laboratories used one of the varieties of serum? uptake methods, and 45 laboratories determined PBI, mostly using the AutoAnalyzer digestor. $\mathrm{A}_{\mathrm{D}}^{\circ}$ few laboratories determined both PBI and thyroxine $\stackrel{?}{?}$ a few determined PBI, thyroxine, and a serum uptak£ test. One laboratory performs all these including thyroxine by both CPB and column methods. Threes 
THYROID TRIAL RESULTS

LABORATORY CODE NO.

\begin{tabular}{|c|c|c|c|c|c|c|}
\hline \multicolumn{2}{|l|}{ Specimen } & $\begin{array}{c}\text { Units } \\
\text { Please } \\
\text { state }\end{array}$ & 1 & 2 & 3 & Method and/or technique used \\
\hline \multicolumn{7}{|l|}{ PBI $\mu \mathrm{g} / 100 \mathrm{ml}$} \\
\hline \multirow{2}{*}{$T_{3}$ Uptake Test } & a) $\frac{\text { Test }}{\text { Standard }}$ & & & & & \\
\hline & b) Standard & & & & & \\
\hline \multicolumn{7}{|c|}{ Thyroxine (by competitive binding) } \\
\hline \multicolumn{7}{|c|}{ Thyroxine (column methods) } \\
\hline Any other test(s) & lease specify & & & & & \\
\hline
\end{tabular}

*A guide to the type of information is: Manual digestion followed by chemistry on AutoAnalyzer. automated digestion and chemistry on AutoAnalyzer, resin uptake-Oxford kit, Thyopac, etc.

INTERPRETATION

\begin{tabular}{|l|l|l|l|}
\hline Specimen & 1 & 2 & 3 \\
\hline PBI & & & \\
\hline$T_{3}$ Uptake Test & & \\
\hline $\begin{array}{c}\text { Thyroxine (by competitive } \\
\text { binding) }\end{array}$ & & \\
\hline Thyroxine (column methods) & & \\
\hline Other tests as specified above) & & & \\
\hline
\end{tabular}

* Please allocate EACH RESULT into that category which is most appropriate using the key and abbreviations below. Please DO NOT attempt to include overall interpretation.

Definite hyperthyroid $\quad H$ Borderline hyperthyroid BH Definite euthyroid E

Borderline hypothyroid BM Definite hypothyroid $\mathbf{M}$

Fig 1 Circular sent out to laboratories participating in the thyroid function test survey

\section{Method}

No. of Laboratories

PBI

AutoAnalyzer digestion and colorimetry

Manual digestion, AutoAnalyzer colorimetry

Manual digestion and colorimetry

33
6
6

Thyroxine

Ames tetralute

Abbott tetrasorb

Biorad

Murphy (1964)

Oxford

Resomat $T_{4}$

(CPB)

(CPB)

(Column)

(CPB)

(Column)

Anon

(CPB)

(Column)

33
6

6

$T_{3}$ Uptake Test Group

Abbott triosorb

Charcoal uptake

Clark and Horn (1965

Resomat 3

Thyopac 3

Total number of laboratories in the survey

Table I Methods used by laboratories in the survey ${ }^{1}$

${ }^{1} A$ few laboratories used more than one method for the same determination laboratories used the Mallinckrodt effective thyroxine ratio kit.

As far as we know there were only two technical problems due to the survey itself. One laboratory that determines thyroxine by a method employing an ethanolic extract which is evaporated to dryness and then redissolved, found the commercial sera difficult to redissolve, though they had no problem with patients' sera. Since most laboratories now use methods where the ethanolic extract is not evaporated to dryness, it is not clear how common a problem this might be. Another laboratory using a column method for measuring thyroxine routinely estimated iodine in two elutions from the column and compared the ratio as an index of contamination. They found abnormally high ratios in the first two sera, suggesting contamination.

In the returns from three laboratories, inspection of all three results suggests that the sera might have been mixed up or incorrectly labelled, so that all three results appear as outliers (see for example figs 2 and 3 ). We did not feel that we should exclude 


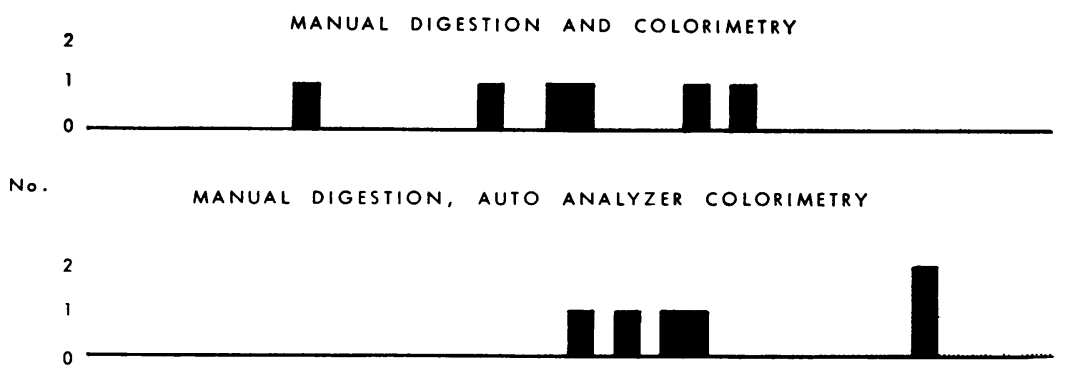

Fig 2 Distribution of results for PBI methods on serum 1

auto analyzer digestion and colorimetry

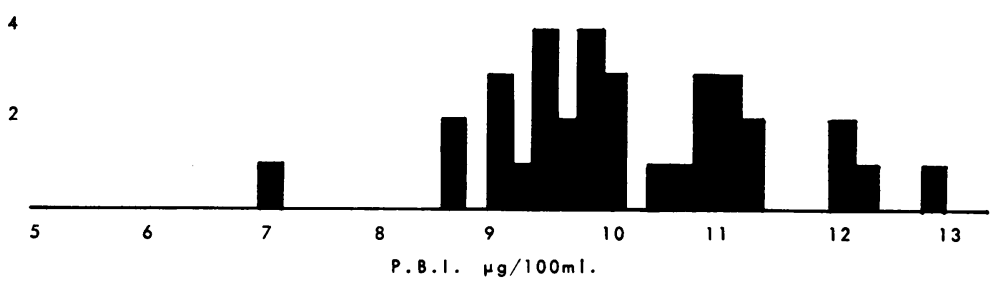

these results subjectively, but instead have calculated means and SDs on all results, and again after excluding those that fell outside \pm 2 SD.

The results, with mean and standard deviation and coefficient of variation, are summarized in table II. The column methods employed for thyroxine determination are very similar in principle and, as there appeared to be no significant difference between the individual groups, we have treated them as one group. Results from competitive proteinbinding methods for thyroxine used by fewer than five laboratories have been excluded. In the serum uptake test group numbers were too small and methods of determination or calculation too diverse for any meaningful grouping or statistics except for Thyopac-3.
The interpretation of tests proved less interesting than we had hoped, with scatter similar to concentrations. The only serious misunderstanding occurred with a serum uptake test, one laboratory reporting a low Thyopac-3 value as hypothyroid, though other values on this serum were correctly interpreted as hyperthyroid.

\section{Discussion}

Firstly, how satisfactory were the freeze-dried commercial sera used in the survey? How stable were they, how precisely dispensed initially, how easy to reconstitute? The results for Thyopac-3, for which the $\mathrm{CV}$ for the three sera were respectively $3 \cdot 9,2 \cdot 6$, and $3 \cdot 1 \%$, indirectly suggest that the sera

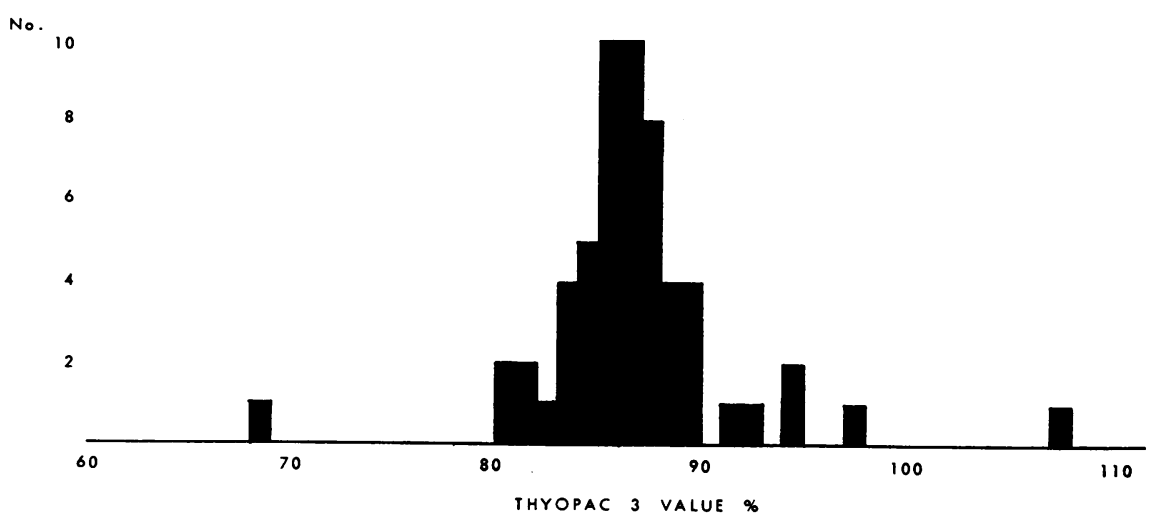

Fig 3 Distribution of results for serum uptake test by Thyopac-3 on serum 2 


\begin{tabular}{|c|c|c|c|c|c|c|c|c|c|c|c|c|}
\hline & \multicolumn{4}{|l|}{ Serum 1} & \multicolumn{4}{|c|}{ Serum 2} & \multicolumn{4}{|l|}{ Serum 3} \\
\hline & Mean & $S D$ & $C V$ & $n$ & Mean & $S D$ & $C V$ & $n$ & Mean & $S D$ & $C V$ & $n$ \\
\hline $\begin{array}{l}\text { PBI as } n m o l / l(\mu \mathrm{g} / 100 \mathrm{ml}) \\
\quad \text { AutoAnalyzer digestion and colorimetry }\end{array}$ & $\begin{array}{l}801 \\
(10 \cdot 17)\end{array}$ & $\begin{array}{l}78 \\
(0 \cdot 99)\end{array}$ & $9 \cdot 7$ & 31 & $\begin{array}{l}615 \\
(7 \cdot 81)\end{array}$ & $\begin{array}{l}50 \\
(0 \cdot 64)\end{array}$ & $8 \cdot 2$ & 31 & $\begin{array}{l}445 \\
(5 \cdot 65)\end{array}$ & $\begin{array}{l}58 \\
(0 \cdot 73)\end{array}$ & $12 \cdot 9$ & 31 \\
\hline Manual digestion, automated colorimetry & $\begin{array}{l}831 \\
(10 \cdot 55)\end{array}$ & $\begin{array}{l}110 \\
(1 \cdot 39)\end{array}$ & $13 \cdot 2$ & 6 & $\begin{array}{l}711 \\
(9 \cdot 02)\end{array}$ & $\begin{array}{l}114 \\
(1 \cdot 45)\end{array}$ & $16 \cdot 1$ & 6 & $\begin{array}{l}516 \\
(6 \cdot 55)\end{array}$ & $\begin{array}{l}117 \\
(1 \cdot 48)\end{array}$ & $22 \cdot 6$ & 2 \\
\hline Manual digestion and colorimetry & $\begin{array}{l}719 \\
(9 \cdot 12)\end{array}$ & $\begin{array}{l}110 \\
(1 \cdot 39)\end{array}$ & $15 \cdot 2$ & 6 & $\begin{array}{l}553 \\
(7 \cdot 02)\end{array}$ & $\begin{array}{l}104 \\
(1 \cdot 32)\end{array}$ & $18 \cdot 8$ & 5 & $\begin{array}{c}390 \\
(4 \cdot 95)\end{array}$ & $\begin{array}{l}65 \\
(0 \cdot 82)\end{array}$ & $16 \cdot 6$ & 6 \\
\hline $\begin{array}{l}\text { Thyroxine by column as nmol } T_{4} \text { iodine } / \\
\quad\left(\mu \mathrm{g} T_{4} \text { iodine } / 100 \mathrm{ml}\right)\end{array}$ & $\begin{array}{l}608 \\
(7 \cdot 71)\end{array}$ & $\begin{array}{l}52 \\
(0 \cdot 66)\end{array}$ & $8 \cdot 6$ & 16 & $\begin{array}{l}507 \\
(6 \cdot 44)\end{array}$ & $\begin{array}{l}41 \\
(0 \cdot 52)\end{array}$ & $8 \cdot 1$ & 16 & $\begin{array}{l}406 \\
(5 \cdot 15)\end{array}$ & $\begin{array}{l}44 \\
0 \cdot 56)\end{array}$ & $10 \cdot 9$ & 15 \\
\hline $\begin{array}{l}C P B \text { methods as nmol } T_{4} \text { iodine } / l\left(\mu \mathrm{g} T_{4} \text { iodine } / 100 \mathrm{ml}\right) \\
\text { Abbott tetrasorb }\end{array}$ & $\begin{array}{l}728 \\
(9 \cdot 24)\end{array}$ & $\begin{array}{l}191 \\
(2 \cdot 43)\end{array}$ & $26 \cdot 3$ & 5 & $\begin{array}{l}526 \\
(6 \cdot 68)\end{array}$ & $\begin{array}{l}197 \\
(2 \cdot 50)\end{array}$ & $37 \cdot 4$ & 5 & $\begin{array}{l}375 \\
(4 \cdot 76)\end{array}$ & $\begin{array}{l}104 \\
(1 \cdot 32)\end{array}$ & $27 \cdot 7$ & 5 \\
\hline Murphy method & $\begin{array}{l}856 \\
(10 \cdot 86)\end{array}$ & $\begin{array}{l}204 \\
(2 \cdot 59)\end{array}$ & $23 \cdot 8$ & 5 & $\begin{array}{l}583 \\
(7 \cdot 40)\end{array}$ & $\begin{array}{l}132 \\
(1 \cdot 67)\end{array}$ & $22 \cdot 6$ & 5 & $\begin{array}{l}350 \\
(4 \cdot 44)\end{array}$ & $\begin{array}{l}108 \\
(1 \cdot 37)\end{array}$ & 30.9 & 5 \\
\hline Thyopac-4 & $\begin{array}{l}915 \\
(11 \cdot 61)\end{array}$ & $\begin{array}{l}80 \\
(1 \cdot 02)\end{array}$ & 8.8 & 42 & $\begin{array}{l}706 \\
(8.96)\end{array}$ & $\begin{array}{l}76 \\
(0.97)\end{array}$ & $10 \cdot 8$ & 42 & $\begin{array}{l}428 \\
(5 \cdot 43)\end{array}$ & $\begin{array}{l}84 \\
(1 \cdot 07)\end{array}$ & $19 \cdot 7$ & 38 \\
\hline $\begin{array}{l}\text { Serum Uptake Tests } \\
\text { Thyopac-3 }\end{array}$ & $67 \cdot 21$ & 2.60 & 3.9 & 56 & $85 \cdot 86$ & $2 \cdot 27$ & 2.6 & 50 & $102 \cdot 63$ & $3 \cdot 23$ & $3 \cdot 1$ & 51 \\
\hline
\end{tabular}

Table II Summary of results on the three sera expressed as the mean coefficient of variation $(C V)$ and number of results ${ }^{1}$

${ }^{1}$ To exclude outliers, all results in each group were calculated first. If any results fell outside mean \pm 2 SD these results were excluded, and mean and SD recalculated from the remaining results.

must have been satisfactory for such excellent agreement to have been possible. The three examples of possibly transposed sera remind us that the most serious errors may be clerical or administrative. Although the numbers of determinations are sometimes small, some suggestive differences in mean values are apparent. To simplify comparisons, observed values for thyroxine have been expressed as thyroxine iodine, though we strongly favour reporting as thyroxine. As expected, values for the column methods are lower than for protein-bound iodine. This difference is more marked for serum 1 and serum 2 than for serum 3, which would tend to support the laboratory which observed that these sera were contaminated. Curiously, there are systematic differences between the results for the three methods of determining PBI. For all three sera, the observed value for manual digestion with automated colorimetry is highest, those for manual digestions and manual colorimetry lowest, with the results for the fully automated method in between.

In a survey of this type it is not possible to decide the true value for thyroxine iodine. The mean values for sera 1 and 2 by Thyopac- 4 are higher than those by other CPB methods or by column, and even higher than PBI. It is not clear why this should be. Work in our laboratory has shown that for the Thyopac- 4 method the thyroxine concentration is, as claimed, approximately proportional to the reciprocal of the radioactivity of the supernatant fluid but that this relationship is not strictly linear, and we have found that this departure from linearity can lead to an overestimate of about $80-95 \mathrm{nmol} / 1$ of thyroxine (1 to $\left.1.2 \mu \mathrm{g} \mathrm{T}_{4} \mathrm{I} / 100 \mathrm{ml}\right)$. This might explain part of the differences, but does not explain why agreement for serum 3 is much closer. Kit methods include serum 'standards'. Watson et al (Watson, Lees and Stafford, 1974) have studied standardization in detail and shown that significant errors can be found in commercial serum standards.

Values for Thyopac-3 show no particular trend and appear excellent for all three sera. For PBI and for thyroxine by Thyopac-4 or column methods the $\mathrm{CV}$ is reasonable at elevated or borderline raised thyroxine concentrations but less satisfactory at normal levels.

Since this is the first time all these thyroid function tests have been surveyed it is difficult to know what counts as an acceptable performance. As a guess we thought that a CV of $10 \%$ or less would be satisfactory. Results for Thyopac-3 were excellent; results for PBI by the fully automated method and for thyroxine by column methods were reasonably satisfactory. Results for manual methods of measuring PBI showed a higher scatter. Thyroxine by the Thyopac-4 method was determined satisfactorily on serum 1 and serum 2 , but there was much greater scatter for the serum with normal thyroxine concentration.

For comparison the $\mathrm{CV}$ for some simpler determinations in the National Quality Control Scheme are shown in table III. These values are from the 'best' group of laboratories, ie, with the largest workload, and lowest variance index, with no exclusions. It is clear that performance of thyroid function tests in vitro by some methods can stand 


\begin{tabular}{lc}
\hline & Coefficient of Variation $(\%)$ \\
\hline Calcium & $3 \cdot 77$ \\
Cholesterol & $8 \cdot 42$ \\
Creatinine & $12 \cdot 40$ \\
Glucose & $8 \cdot 08$ \\
Potassium & $3 \cdot 03$ \\
Sodium & $1 \cdot 59$ \\
Tota! protein & $4 \cdot 07$ \\
Urea & $5 \cdot 49$ \\
Urate & $8 \cdot 62$ \\
\hline
\end{tabular}

Table II Interlaboratory variation for routine determinations in clinical chemistry based on data from the National Quality Control Scheme covering 10 consecutive results from laboratories with workloads exceeding 250000 tests per annum

comparison with that for cholesterol, creatinine, glucose, and urate. As microgram rather than milligram quantities are being analysed, this is a fair achievement, though in view of the diagnostic importance of thyroid function tests there is considerable room for improvement.

In competitive protein-binding methods analytical errors are magnified alarmingly by the shape of the standard curve. For example, in the Thyopac-4 method a $2 \%$ overall error in the technical procedures including extraction, pipetting, and counting, would lead to very much more than a $2 \%$ error in the measured thyroxine. The absolute error is similar throughout the range, so that proportionately it is much worse at low thyroxine concentration. For example, at a true thyroxine concentration of $51.5 \mathrm{nmol} / \mathrm{l}(4 \mu \mathrm{g} / 100 \mathrm{ml})$ a $2 \%$ methodological error could give values from $41-62 \mathrm{nmol} / 1 \mathrm{(3 \cdot 2}$ to $4.8 \mu \mathrm{g} / 100 \mathrm{ml}$, or up to $20 \%$ error. In the middle of the range, $2 \%$ error in counts would lead to about 8 to $10 \%$ error in results. Since the error in counting alone is rarely much less than $1 \%$, the extraction and subsequent pipettings make considerable demands on technical skill.

The reporting of the serum uptake group of tests is very confusing for the unwary, with the ridiculous situation of two opposite conventions of expressing results quite apart from different analytical methods. Since the test is an empirical one, it does not matter unduly which convention is used. We would prefer to calculate the index so that high values occurred in thyrotoxicosis and low values in hypothyroidism ${ }^{1}$, but any agreed convention would be better than the present confusion. In general there seemed good agreement in interpreting thyroxine values. The Radiochemical Centre booklet for Thyopac-4 however quoted a normal range of approximately

\footnotetext{
${ }^{1}$ The reverse is recommended in the first contribution on 'Nomenclature' as the test measures unsaturated binding sites which are typically high in hypothyroidism and low in hyperthyroidism.-ED.
}

$59-170 \mathrm{nmol} / \mathrm{l}\left(4.6\right.$ to $13.0 \mu \mathrm{g} / 100 \mathrm{ml}$ as thyroxine) $\stackrel{\overrightarrow{\mathrm{O}}}{\frac{\overrightarrow{\mathrm{O}}}{\mathrm{a}}}$ Several laboratories interpreted values in the $150-155 \mathrm{nmol} / 1 / 11.6$ to $12.0 \mu \mathrm{g} / 100 \mathrm{ml})$ region as? borderline hyperthyroid; this fits in with our own experience, which is that the upper limit of normal i은 around $140-155 \mathrm{nmol} / \mathrm{l}(11 \cdot 0$ to $12 \cdot 0 \mathrm{ug} / 100 \mathrm{~m} \mu)$.

\section{Conclusions}

After one survey any conclusions must, obviously $\vec{\circ}$ be tentative; nevertheless, it would appear (1) that methods for measuring PBI other than by a fully automatic method are no longer acceptable; ( $2 \overrightarrow{8}$ there is a need for commercial control serum of highi quality, standardized by the most widely usec methods in this country; (3) competitive proteinfu binding methods for thyroxine need improvement to ensure better reproducibility, especially at low? thyroxine concentrations.

No firm conclusions should be drawn about the relative merits of commercially available kits since $\frac{0}{\circ}$ where numbers are small, one set of aberrane results may greatly increase the coefficient of의 variation for the whole group. Watson and Leeșr (1973) recently compared some available kits for thyroxine assay.

The results of this preliminary survey suggest thae though kits can give excellent results, eg, Thyopac-3 simply following instructions in an otherwisep satisfactory kit is not enough to ensure satisfactory results. It is a misconception that because a kit is commercially available from a reputable manu facturer, and the method is apparently simple tof perform, any laboratory can complete the determina tion satisfactorily. Great vigilance in quality contros is still necessary. It is planned in the near future to introduce regular surveys of in vitro tests of thyroiep. function through the National Quality ControB Scheme.

\section{References}

Clark, F., and Horn, D. B. (1965). Assessment of thyroid function by the combined use of the serum protein bound iodine and resinfo uptake of ${ }^{131}$ I-triiod othyronine. J. clin. Endocr., 25, 39-45.

Murphy, B. E. P., and Pattee, C. J. (1964). Determination of thyroxind utilizing the property of protein binding. J. clin. Endocr., $24 \mathrm{~N}$ 187-196.

Watson, D., and Lees, S. (1973). Comparative study of thyroxino assays employing kit radio-ligand reagents. Ann. clin. Biochem., 10, 14-22.

Watson, D., Lees, S., and Stafford, J. E. H. (1974). Accuracy an standardisation of serum thyroxine assays. Ann. clin. Biochem. 11, 1-7.

Whitehead, T. P., Browning, D. M., and Gregory, A. (1973). AD comparative survey of the results of analyses of blood serum iro clinical chemistry laboratories in the United Kingdom. $J \vec{D}^{+}$ clin. Path., 26, 435-445.

Whitehead, T. P., and Morris, L. O. (1969). Methods of quality control. Ann. clin. Biochem., 6, 94-103. 